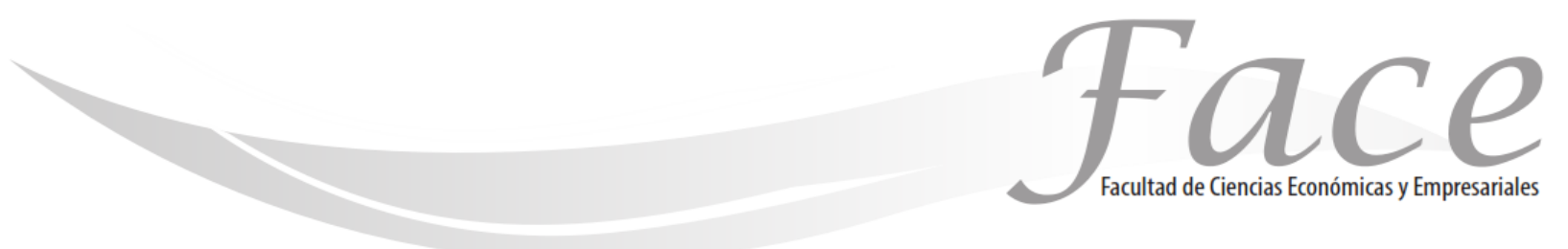

ISSN Impreso: 1794-9920

ISSN Electrónico: 2500-9338

Volumen 19-N¹

Año 2019

Págs. 109 - 121

(c) (i) $\odot$

\title{
ESTRATEGIAS DE DIFERENCIACIÓN PARA INCREMENTAR LA CALIDAD EN INSTITUCIONES DEL SECTOR SALUD DE TABASCO.
}

\author{
Miguel Ángel Ramírez Martínez* \\ ORCID: https://orcid.org/0000-0001-7131-1066
}

Oscar Priego Hernández **

Fecha de Recepción: Febrero 5 de 2019

Fecha de Aprobación: Mayo 31 de 2019

\section{Resumen:}

La salud pública es un factor determinante de la riqueza de las naciones del siglo XXI. A pesar de la importancia de la salud, hasta la fecha no se encontraron estudios en el estado de Tabasco, que reflejen las características de los usuarios de los diferentes hospitales y clínicas del sector público y el estado mexicano ha generado el Programa Sistema Integral de Calidad en Salud para medir la calidad de los servicios. Objetivo: Medir la calidad del servicio que se ofrece en el sector salud. Materiales y método: Se trata de un estudio exploratorio que trata un tema poco estudiado desde el enfoque de la mercadotecnia en el estado de Tabasco, nos referiremos a hospitales y clínicas del sector público quienes brinda atención a 364,055 derecho habientes de Villahermosa. La muestra resultante fue de 755 , aplicándose el cuestionario finalmente a 930, para la captura y procesamiento de los cuestionarios se utilizó el paquete estadístico Statistical Package for Social Sciences, versión 21. El trabajo identificó dos aspectos: En primer lugar las características generales del paciente y en segundo lugar, la calidad de los servicios, evaluados a través del modelo SERVQUAL.Resultados: Muestran que la mayoría de los pacientes tiene un grado de estudios de licenciatura y que más del $50 \%$ se encuentra entre los 26 y 45 años, así como que la ineficiencia del personal, incumplo con la entrega de documentos, tramites a tiempo y la insatisfacción de los usuarios con el servicio recibido son los elementos que resultaron con mayor deficiencias para explicar la buena calidad en el servicio. Conclusiones: Se proponen estrategias de producto y de diferenciación para alcanzar dicha calidad y de esta manera se cumpla con la misión de este tipo de instituciones.

Palabras clave: marketing político, presidente ideal, distancia de Hamming, subconjunto borroso.

\footnotetext{
*Profesor-Investigador, Dacea-Ujat - Centro de Investigaciones - Universidad Juárez Autónoma de Tabasco- México. Contacto: mangel113@hotmail.com

** Investigador. - Universidad Juárez Autónoma de Tabasco- México. Contacto: priegooscarh@hotmail.com
} 


\title{
DIFFERENTIATION STRATEGIES TO INCREASE QUALITY IN INSTITUTIONS OF THE HEALTH SECTOR OF TABASCO.
}

\begin{abstract}
:
Public health is a determining factor in the wealth of the nations of the 21st century. Despite the importance of health, to date no studies were found in the state of Tabasco that reflect the characteristics of the users of the different hospitals and clinics of the public sector and the Mexican State has generated the Comprehensive Quality System Program in Health to measure the quality of services. Objective: To measure the quality of the service offered in the health sector. Materials and methods: This is an exploratory study that deals with a subject little studied from the marketing approach in the state of Tabasco, we will refer to hospitals and public sector clinics who provide care to 364,055 Villahermosa law holders. The resulting sample was 755 , the questionnaire finally being applied to 930 , for the capture and processing of the questionnaires the statistical package Statistical Package for Social Sciences, version 21 was used. The work identified two aspects: First, the general characteristics of the patient and second, the quality of the services, evaluated through the SERVQUAL model. Results: They show that the majority of patients have a bachelor's degree and that more than $50 \%$ are between 26 and 45 years old. as the inefficiency of the staff, failure to deliver documents, timely procedures and the dissatisfaction of users with the service received are the elements that were most deficient to explain the good quality of the service. Conclusions: Product and differentiation strategies are proposed to achieve said quality and in this way the mission of this type of institutions is fulfilled.
\end{abstract}

Keywords: Differentiation strategies; Health; Quality of service; Servqual

\section{ESTRATÉGIAS DE DIFERENCIAÇÃO PARA AUMENTAR A QUALIDADE NAS INSTITUIÇÕES DO SETOR DE SAÚDE DE TABASCO.}

\section{Resumo:}

A saúde pública é um fator determinante na riqueza das nações do século XXI. Apesar da importância da saúde, até o momento não foram encontrados estudos no estado de Tabasco que reflitam as características dos usuários dos diferentes hospitais e clínicas do setor público e o Estado mexicano gerou o Programa Sistema Integral de Qualidade em Saúde para mensurar o qualidade dos serviços. Objetivo: Mensurar a qualidade do serviço oferecido no setor de saúde. Materiais e métodos: Trata-se de um estudo exploratório que trata de um assunto pouco estudado a partir da abordagem de marketing no estado de Tabasco, nos referiremos a hospitais e clínicas do setor público que prestam atendimento a 364.055 advogados de Villahermosa. A amostra resultante foi de 755 , o questionário foi finalmente aplicado a 930, para a captura e processamento dos questionários foi utilizado o pacote estatístico Statistical Package for the Social Sciences, versão 21. 0 trabalho identificou dois aspectos: primeiro, as características gerais do paciente e, segundo, a qualidade dos serviços, avaliada pelo modelo SERVQUAL. Resultados: Eles mostram que a maioria dos pacientes é bacharel e que mais de $50 \%$ têm entre 26 e 45 anos. como a ineficiência do pessoal, a falta de entrega de documentos, procedimentos oportunos e a insatisfação dos usuários com o serviço recebido são os elementos mais deficientes para explicar a boa qualidade do serviço. Conclusões: Estratégias de produto e diferenciação são propostas para atingir essa qualidade e, desta forma, a missão deste tipo de instituições é cumprida.

Palavras-chave: Estratégias de diferenciação; Saúde; Qualidade de serviço; Servqual 


\section{INTRODUCCIÓN:}

En la segunda década del siglo XX surge en los Estados Unidos los primeros estándares mínimos de actuación profesional que debería cumplir los miembros del Colegio de Cirujanos en hospitales Señalan:

... en los inicios de la década de los 70 s del siglo XX, el interés por evaluar la calidad y la productividad de los sistemas y centros de atención a la salud se incrementó sustancialmente como producto de las políticas de salud impulsadas por la Organización Mundial de la Salud (OMS) y las crecientes restricciones presupuestales para lograr los objetivos diseñados (Salinas y González, 2006).

La organización panamericana de la salud (OPS) en el Perfil del Sistema de Servicios de Salud en México (1998) establece: "La calidad de la atención es un área de desarrollo dentro del sector público y desde 1997 se impulsan líneas de acción como son: en la SSA, establecer un diagnóstico basal en las unidades y a partir del mismo implementar programas de mejoría de la calidad, así como la constitución en hospitales y niveles subnacional de comisiones (de expediente clínico, de mortalidad, de referencia y contra referencia, etc.); en el IMSS, el programa de atención al enfermo crónico en el domicilio, entre otros. En el año 2001 hay un renovado esfuerzo, más amplio, integral y participativo, para mejorar la calidad de los servicios públicos y privados con la

"Cruzada Nacional por la Calidad de los Servicios de Salud que incluye la definición y registro de indicadores de calidad en unidades y la conformación de una red nacional de monitoreo, la capacitación de trabajadores y directivos en áreas que inciden en la calidad de los servicios, la elaboración y divulgación de derechos de los pacientes y de códigos de ética de enfermeras y médicos, así como el otorgamiento de apoyos técnicos y financieros federales a proyectos locales que apuntan a mejorar la calidad asistencial (OPS, 2002).

En el diagnóstico realizado en el programa sectorial de salud 2013-2018 se establece:

"En el período 2007-2012, la Cruzada Nacional por la Calidad migró al Sistema Integral de Calidad en Salud (SICALIDAD), cuya existencia estuvo justificada desde tres perspectivas: la de los usuarios, por la insatisfacción con la calidad otorgada en los servicios; la de los profesionales de la salud, por la alta variabilidad en los procedimientos médicos y la falta de estandarización de los cuidados de enfermería, la deficiencia en los programas académicos para los profesionales, la falta de congruencia entre el financiamiento y los incentivos, inadecuado desempeño de las organizaciones de salud, debido a la falta de integración entre las prioridades de salud y los proyectos, falta de posicionamiento de la calidad a nivel de la alta dirección, lo cual dio como resultado poca respuesta de las organizaciones de salud a la opinión de los usuarios".

Señalando:

EI SICALIDAD tuvo como principal objetivo: situar la calidad como una prioridad en la agenda permanente del Sistema Nacional de Salud" y estableció tres objetivos específicos con una denominación desde los ejes conceptuales de la calidad en salud: la calidad técnica y seguridad del paciente, la calidad percibida por los usuarios y la calidad en la gestión de los servicios de salud (Secretaría de Salud, 2002).

El análisis de la calidad en el servicio se inicia formalmente con el artículo de catedráticos de marketing que realizaron una investigación de la calidad de los servicios en 1985, creando una escala para calificar a las empresas de servicio de acuerdo a cinco dimensiones: tangibilidad, fiabilidad, respuesta, seguridad y empatía, y es a partir de esa investigación que se propusieron un modelo de calidad en el servicio.

El modelo SERVQUAL es una escala multidimensional constituida por dos sub-escalas, una de expectativas y otra de percepciones. Contempla cinco dimensiones de la calidad del servicio. Este modelo evalúa la calidad del servicio a partir de las diferencias entre las expectativas y percepciones de los usuarios, por lo que se considera como el más apropiado para evaluar la calidad del servicio en el sector salud (Parasuraman, Zeithaml y Berry, 1993).

La salud pública es un elemento determínate de la productividad de las poblaciones que habitan en las sociedades del conocimiento, de ella depende el cambio cualitativo y cuantitativo del capital humano de un país, por lo que juega un papel estratégico en el crecimiento y desarrollo de las poblaciones y en la competitividad de las 
naciones. El estado es el encargado de brindar los servicios de salud que permitan a la población poder desarrollar todas sus potencialidades y al mismo tiempo tener una mejor calidad de vida.

De acuerdo a la Constitución Política de los Estados Unidos Mexicanos, en su artículo $4^{\circ}$ establece:

Toda persona tiene derecho a la protección de la salud. La ley definirá las bases y modalidades para el acceso a los servicios de salud y establecerá la concurrencia de la federación y las entidades federativas en materia de salubridad general, conforme a lo que dispone la fracción XVI del artículo 73 de esta constitución (Constitución Política, 2017)

En Tabasco, en el 2011 existían 659 unidades médicas, de las cuales 623 eran de consulta externa y 32 hospitalarias, quienes daban atención a 2, 021,979 usuarios, mientras que en el municipio del Centro existían 115 unidades, las cuales daban atención a 760,909 usuarios (INEGI, 2011). Existen cuatro instituciones de salud pública en Tabasco: Instituto de Seguridad Social del Estado de Tabasco (ISSET), Instituto de Seguridad y Servicios Sociales de los Trabajadores del Estado (ISSSTE), Instituto Mexicano del Seguro Social (IMSS) y Secretaria de Salud-, las cuales están distribuidas en los 17 municipios del estado.En este estudio nos referiremos al conjunto de hospitales y clínicas del sector público, tanto federal como estatal, que proporcionan los servicios de consulta externa y hospitalarias, brindando atención al derecho habiente de la ciudad de Villahermosa, un total de 364,055 usuarios (INEGI, 2011)

Existen una serie de estudios utilizando el modelo SERVQUAL para identificar la calidad del servicio en clínicas y hospitales en varios países, entre los cuales, se encuentran: La calidad de servicio en el sector de salud pública: una investigación empírica realizada en Talxahuano Sur, Chile (Valenzuela, 2008); Calidad percibida del cuidado hospitalario: adaptación del SERVQUAL al hospital público, realizado en Alicante, España, por Mira, Buil, Rodriguez y Aranáz (1997); Calidad de servicio de la consulta externa de medicina interna de un hospital general de Lima mediante la encuesta SERVQUAL realizado por Casalino-Carpio (2008); Evaluación de la calidad del servicio al cliente mediante la aplicación del modelo SERVQUAL en una clínica del sector salud realizado por Rios, Samblé y Reyes (2008) en Ciudad Juárez, Chihuahua, México, todos se enfocan en las cinco dimensiones del modelo y determinan si la calidad es buena o no y dan recomendaciones para que los hospitales y clínicas del sector salud realicen las mejoras que permitan otorgar mejor servicios a sus derechohabientes.
Hasta la fecha no se encontraron estudios en el estado de Tabasco, que reflejen las características de los usuarios de los diferentes hospitales y clínicas del sector público del estado, lo que genera que no haya un política pública que permita establecer estrategias específicas para la atención a sus derechohabientes, generándose una serie de problemas (los cuales tienen causas multifactoriales, pero en ellos incide el desconocimiento de los perfiles de usuarios) como: un desabasto de medicamentos, falta de médicos especialistas y/o falta de espacios para la atención de sus pacientes, que muchas veces se reflejan en la pérdida de vidas humanas, por lo que este estudio permitirá identifica las características (el perfil) de los usuarios-derechohabientes y la calidad de los servicios, evaluados a través del modelo SERVQUAL y se propondrán estrategias que aplicadas podrían mejorar la calidad de los servicios.

Por lo anteriormente señalado el objetivo de esta investigación es medir la calidad del servicio que se ofrece en el sector salud, por lo que nuestras unidades de análisis son los hospitales y clínicas del sector público.Esta investigación solo utiliza un cuestionario (medir percepciones) que distingue dos partes: la primera, consta de siete interrogantes que permiten conocer el perfil del usuario y la segunda de 28 preguntas a través de las cuales se puede conocer las percepciones de los usuarios en relación al servicio. El instrumento está conformado por una escala de respuestas múltiples (escala de Liker) diseñada para comprender las percepciones de los clientes respecto a un servicio. Permite evaluar, pero a su vez es un instrumento de mejora y de comparación con otras organizaciones. Es decir, mide lo que el cliente percibe de la organización que presta el servicio en las cinco dimensiones mencionadas anteriormente.

El plan estratégico de la empresa establece los tipos de negocios que la empresa tendrá en operación y los objetivos de cada uno de ellos. El marketing desempeña un rol fundamental en la planeación estratégica de la empresa de varias formas. Primero, el marketing proporciona una filosofía rectora el concepto de marketing que sugiere que la estrategia de la empresa deberá girar alrededor de la construcción de relaciones redituables con los grupos importantes de clientes; segundo, ayuda a quien hace la planeación estratégica a identificar oportunidades atractivas de mercado y evaluar el potencial de la empresa para aprovecharlas. Por último, dentro de las oportunidades de negocios individuales, el marketing diseña estrategias para llegar a los objetivos de la unidad. El valor del cliente es el ingrediente clave en la fórmula para el éxito del mercadólogo (Kotler, Amstrong, 2013).

Esta estrategia tiene por objeto dotar al producto de cualidades distintivas que los diferencien de la oferta de los competidores. A consecuencia de la lealtad de marca que genera el producto diferenciado se crea una barrera de 
entrada que dificulta el ingreso de nuevos competidores, al exigir a las nuevas empresas entrantes elevados volúmenes de inversión para captar a los clientes satisfechos con la marca establecida. El principal problema que acompaña a esta estrategia es la dificultad para mantener la exclusividad del producto (Munuera y Rodríguez, 2012).

\section{METODOLOGIA:}

Es un estudio de tipo exploratorio, con un enfoque cuantitativo, utilizándose para la recolección de datos un cuestionario (el cual ha sido adaptado para México) que consta de dos secciones: la primera, tiene siete interrogantes que permiten conocer el perfil del usuario y la segunda, integrada por 28 preguntas que arroja las percepciones de los usuarios en relación al servicio.

El universo de estudio en esta investigación son los usuarios/derechohabientes de las clínicas y hospitales del sector público objeto de estudio, que eran 364,055 hasta el 2010. El tamaño de la muestra fue calculado a partir de la siguiente ecuación para poblaciones finitas:

$$
n=\frac{Z_{a}^{2} * \mathrm{~N} * \mathrm{p} * \mathrm{q}}{i^{2}(\mathrm{~N}-1)+Z_{a}^{2} * \mathrm{p} * \mathrm{q}}
$$

Para sustituir los datos en la ecuación tomamos como referencia un Nivel de confianza $\left(Z^{2}\right)$ igual a $1.65(90 \%)$, la población (N) es igual a 364,055 usuarios, la probabilidad a favor (p) es igual a 0.5 , la probabilidad en contra (q) es igual a 0.5 y el error de estimación (i) es igual a 0.03 (3\%). La muestra resultante fue de 755 derechohabientes, aplicándose finalmente a 930 usuarios.

El cuestionario se suministró a los derecho-habientes de las clínicas y hospitales que se localizan en el municipio del centro del estado de Tabasco de enero de 2015 a junio de 2016. Al mismo tiempo se tomó información de tipo cualitativa que no se expone en esta investigación.

Las variables demográficas a medir son; edad, sexo, escolaridad Tipo de servicio que demandan y de que municipio del estado provienen y las dimensiones que se evalúan en relación al servicio son: elementos tangibles, seguridad, empatía, fiabilidad y credibilidad, de acuerdo al modelo utilizado en esta investigación.

La hipótesis de esta investigación es que la calidad del servicio de las clínicas y hospitales públicos de Villahermosa, capital del estado de Tabasco, es mala o de baja calidad.

\section{RESULTADOS:}

Los resultados obtenidos de la información arrojada por los cuestionarios y analizada a través del SPSS Versión 21, son los siguientes:

\section{Perfil del usuario.}

El estudio arroja que existe una mayor frecuencia de usuarios del género femenino que acuden 0 utilizan el servicio del hospital ya que el 66.78 es del género femenino y un $33.22 \%$ del género masculino.

La frecuencia de usuarios por escolaridad señalan que el $0.07 \%$ de los usuarios no tiene ninguna, el $3.2 \%$ no terminó la primaria, el $6.7 \%$ terminó la Primaria, el $15.6 \%$ la Secundaria, el $23.5 \%$ el Bachillerato, el $24.7 \%$ la licenciatura y el $24.8 \%$ tienen maestría, es decir, hay una frecuencia mayor de usuarios con estudios de maestría que utilizan el servicio.

En cuanto a la edad de los entrevistados, que en algunos casos no fueron los pacientes o usuarios, los datos señalan que el $21.9 \%$ de los usuarios son menores de 25 años, el $30.64 \%$ tiene de 26 a 35 años, el otro $24.51 \%$ tiene de 36 a 45 años y el $22.90 \%$ tiene más de 45 años, es decir, hay una frecuencia mayor de usuarios de 26 a 35 años de edad que utilizan el servicio.

El $51.8 \%$ de los usuarios utilizan el servicio de consulta externa, el $14.8 \%$ el servicio de urgencias, el $7.4 \%$ el servicio de laboratorio y el $26 \%$ de los usuarios el servicio de hospitalización, es decir, hay una frecuencia mayor de usuarios que utilizan el servicio de consulta externa.

Tabla 1: Frecuencia por servicio solicitado.

\begin{tabular}{l|c|c}
\hline $\begin{array}{l}\text { Servicio } \\
\text { solicitado }\end{array}$ & Frecuencia & Porcentaje \\
\hline $\begin{array}{l}\text { Consulta } \\
\text { externa }\end{array}$ & 482 & 51.8 \\
\hline Urgencias & 138 & 14.8 \\
\hline Laboratorio & 69 & 7.4 \\
\hline Hospitalización & 241 & 26.0 \\
\hline Total & 930 & $100 \%$ \\
\hline
\end{tabular}

Nota: Elaboración propia.

De acuerdo al Municipio donde radican los usuarios que acuden a los hospitales y clínicas públicos, se observa que la mayor frecuencia de estos proviene del municipio del Centro, seguido por Cárdenas, Nacajuca, Comalcalco, y también vienen de municipios del Estado de Chiapas. 
ISSN: 1794-9920 Impreso / Electrónico 2500-9338

Enero - Julio de 2019 - Volumen 19 Número 2, Año 2019 Págs. 109 - 121

\section{Percepciones y experiencias de los usuarios y/o} pacientes por dimensión.

Los resultados obtenidos de la segunda parte del cuestionario aplicado mide la calidad del servicio en función de las cinco variables del modelo utilizado, obteniéndose los siguientes datos:

\section{Elementos tangibles.}

Se refiere a la apariencia del personal, facilidades físicas, equipo e información con el cual cuenta el hospital, los usuarios-derechohabientes los califican como malo y evalúan como buena la apariencia aseada y ordenada de los empleados del hospital, la correlación bilateral es significativa al nivel 0,01.
En la dimensión de Elementos Tangibles, con excepción del ítem ET_27, los demás muestran la baja calidad de la clínica u hospital y está fuertemente asociada al grado de insatisfacción por la calidad del servicio recibido por los clientes en las clínicas y hospitales en el municipio del Centro, Tabasco. (Tabla 2).

Tabla 2: Coeficiente Rho de Sperman en forma global y por ítem para la dimensión de Elementos Tangibles

\begin{tabular}{|c|c|c|c|c|c|c|c|c|c|c|c|}
\hline \multicolumn{3}{|c|}{ COEFICIENTES } & GGLOB & ET-1 & ET-2 & ET-3 & ET-4 & ET-25 & ET-26 & ET-27 & ET-28 \\
\hline & \multirow[t]{3}{*}{ GGLOB } & Coeficiente de correlación & 1.000 & $.715^{* *}$ & $.654^{* *}$ & $.719^{* *}$ & $.613^{* *}$ & $.764^{* *}$ & $.717^{* *}$ & $.766^{* *}$ & $.774^{* *}$ \\
\hline & & Sig. (bilateral) & & .000 & .000 & .000 & .000 & .000 & .000 & .000 & .000 \\
\hline & & $\mathrm{N}$ & 1860 & 930 & 930 & 930 & 930 & 930 & 930 & 930 & 930 \\
\hline & \multirow[t]{3}{*}{ ET-1 } & Coeficiente de correlación & $.715^{* *}$ & 1.000 & $.757^{*+}$ & $.614^{* *}$ & $.506^{* *}$ & $.497^{*+}$ & $.395^{* *}$ & $.515^{* *}$ & $.484^{* *}$ \\
\hline & & Sig. (bilateral) & .000 & & .000 & .000 & .000 & .000 & .000 & .000 & .000 \\
\hline & & $\mathrm{N}$ & 930 & 930 & 930 & 930 & 930 & 930 & 930 & 930 & 930 \\
\hline & \multirow[t]{3}{*}{ ET-2 } & Coeficiente de correlación & $.654^{*+}$ & $.757^{*+}$ & 1.000 & $.645^{* *}$ & $.432^{* *}$ & $.461^{* *}$ & $.377^{* *}$ & $.440^{* *}$ & $.419^{* *}$ \\
\hline & & Sig. (bilateral) & .000 & .000 & & .000 & .000 & .000 & .000 & .000 & .000 \\
\hline & & $\mathrm{N}$ & 930 & 930 & 930 & 930 & 930 & 930 & 930 & 930 & 930 \\
\hline & \multirow[t]{3}{*}{ ET-3 } & Coeficiente de correlación & $.719^{*+}$ & $.614^{* *}$ & $.645^{* *}$ & 1.000 & $.582^{* *}$ & $.560^{* *}$ & $.467^{* *}$ & $.475^{* *}$ & $.507^{* *}$ \\
\hline & & Sig. (bilateral) & .000 & .000 & .000 & & .000 & .000 & .000 & .000 & .000 \\
\hline & & $\mathrm{N}$ & 930 & 930 & 930 & 930 & 930 & 930 & 930 & 930 & 930 \\
\hline & \multirow[t]{3}{*}{ ET-4 } & Coeficiente de correlación & $.613^{* *}$ & $.506^{* *}$ & $.432^{* *}$ & $.582^{* *}$ & 1.000 & $.453^{* *}$ & $.415^{* *}$ & $.379^{*+*}$ & $.421^{* *}$ \\
\hline & & Sig. (bilateral) & .000 & .000 & .000 & .000 & & .000 & .000 & .000 & .000 \\
\hline & & $\mathrm{N}$ & 930 & 930 & 930 & 930 & 930 & 930 & 930 & 930 & 930 \\
\hline & \multirow[t]{3}{*}{ ET-25 } & Coeficiente de correlación & $.764^{* *}$ & $.497^{* *}$ & $.461^{*}$ & $.560^{* *}$ & $.453^{* *}$ & 1.000 & $.631^{* *}$ & $.614^{* *}$ & $.657^{* *}$ \\
\hline & & Sig. (bilateral) & .000 & .000 & .000 & .000 & .000 & & .000 & .000 & .000 \\
\hline & & $\mathrm{N}$ & 930 & 930 & 930 & 930 & 930 & 930 & 930 & 930 & 930 \\
\hline & \multirow[t]{3}{*}{ ET-26 } & Coeficiente de correlación & $.717^{*+}$ & $.395^{* *}$ & $.377^{*+}$ & $.467^{* *}$ & $.415^{* *}$ & $.631^{* *}$ & 1.000 & $.762^{*+*}$ & $.778^{* *}$ \\
\hline & & Sig. (bilateral) & .000 & .000 & .000 & .000 & .000 & .000 & & .000 & .000 \\
\hline & & $\mathrm{N}$ & 930 & 930 & 930 & 930 & 930 & 930 & 930 & 930 & 930 \\
\hline & \multirow[t]{3}{*}{ ET-27 } & Coeficiente de correlación & $.766^{* *}$ & $.515^{* *}$ & $.440^{* *}$ & $.475^{* *}$ & $.379^{* *}$ & $.614^{* *}$ & $.762^{* *}$ & 1.000 & $.838^{* *}$ \\
\hline & & Sig. (bilateral) & .000 & .000 & .000 & .000 & .000 & .000 & .000 & & .000 \\
\hline \multirow{4}{*}{ Rho de Spearman } & & $\mathrm{N}$ & 930 & 930 & 930 & 930 & 930 & 930 & 930 & 930 & 930 \\
\hline & \multirow[t]{3}{*}{ ET-28 } & Coeficiente de correlación & $.774^{* *}$ & $.484^{* *}$ & $.419^{* *}$ & $.507^{* *}$ & $.421^{* *}$ & $.657^{* *}$ & $.778^{* *}$ & $.838^{* *}$ & 1.000 \\
\hline & & Sig. (bilateral) & .000 & .000 & .000 & .000 & .000 & .000 & .000 & .000 & \\
\hline & & $\mathrm{N}$ & 930 & 930 & 930 & 930 & 930 & 930 & 930 & 930 & 930 \\
\hline
\end{tabular}

**. La correlación es significativa al nivel 0,01 (bilateral).

Nota: Elaboración propia. 


\section{Fiabilidad.}

Describe la habilidad que tienen los prestadores del servicio para proporcionar el servicio prometido con precisión y eficacia a los usuarios, los derechohabientes califican el grado de calidad del servicio como regular, lo que significa que la prestación del servicio no es el que espera el cliente, la correlación bilateral es significativa al nivel 0,01. En la dimensión de Fiabilidad, solamente el ítem Fl-7 muestra una valoración buena por los clientes; esta se refiere a que el hospital brinda la primera vez, un buen servicio y está fuertemente asociada al grado de satisfacción de la calidad del servicio recibido por los clientes en las clínicas y hospitales en el municipio del Centro, Tabasco (Tabla 3).

Tabla 3: Coeficiente Rho de Sperman en forma global y por ítem para la dimensión de Fiabilidad

\section{Correlaciones}

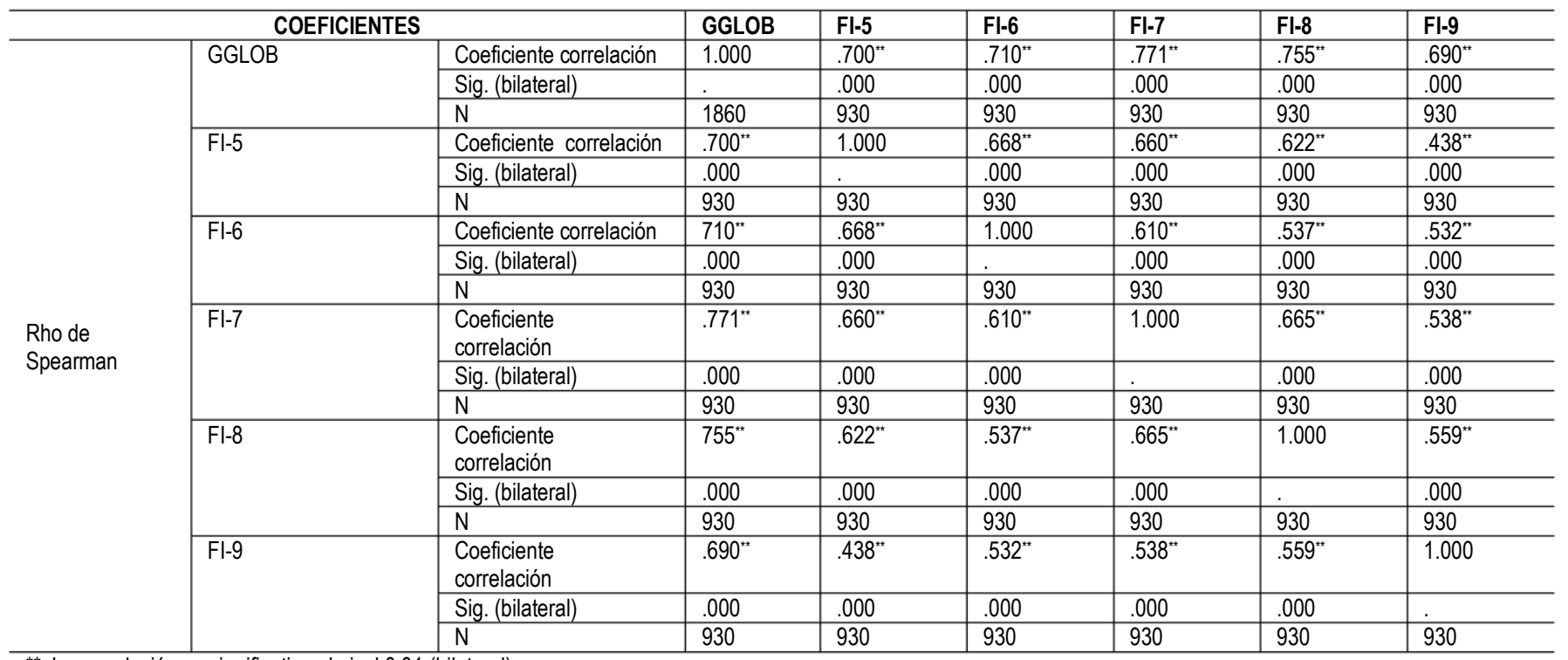

\footnotetext{
**. La correlación es significativa al nivel 0,01 (bilateral).
}

Nota: Elaboración propia.

Credibilidad.

Representa la habilidad que tienen los prestadores del servicio para ayudar y proporcionar el servicio rápidamente a los usuarios, los derechohabientes califican el grado de calidad del servicio como regular, la correlación bilateral es significativa al nivel 0,01.

En esta dimensión, solo el ítem CR-14 refleja una aceptación buena por parte de los usuarios (Tabla 4) 
ISSN: 1794-9920 Impreso / Electrónico 2500-9338

Enero - Julio de 2019 - Volumen 19 Número 2, Año 2019 Págs. 109 - 121

Tabla 4: Coeficiente Rho de Sperman en forma global y por ítem para la dimensión de Credibilidad

\section{Correlaciones}

\begin{tabular}{|c|c|c|c|c|c|c|c|c|}
\hline \multicolumn{3}{|c|}{ COEFICIENTES } & GGLOB & CR-10 & CR-11 & CR-12 & CR-13 & CR-14 \\
\hline & \multirow[t]{3}{*}{ GGLOB } & $\begin{array}{l}\text { Coeficiente de } \\
\text { correlación }\end{array}$ & 1.000 & $.691^{* *}$ & $.756^{* *}$ & $.742^{* *}$ & $.636^{* *}$ & $.789^{*+}$ \\
\hline & & Sig. (bilateral) & & .000 & .000 & .000 & .000 & .000 \\
\hline & & $\mathrm{N}$ & 1860 & 930 & 930 & 930 & 930 & 930 \\
\hline & \multirow[t]{3}{*}{ CR-10 } & $\begin{array}{l}\text { Coeficiente de } \\
\text { correlación }\end{array}$ & $.691^{* *}$ & 1.000 & $.555^{* *}$ & $.516^{* *}$ & $.421^{* *}$ & $.552^{*+*}$ \\
\hline & & Sig. (bilateral) & .000 & & .000 & .000 & .000 & .000 \\
\hline & & $\mathrm{N}$ & 930 & 930 & 930 & 930 & 930 & 930 \\
\hline & \multirow[t]{3}{*}{ CR-11 } & $\begin{array}{l}\text { Coeficiente } \\
\text { correlación }\end{array}$ & $.756^{*+}$ & $.555^{* *}$ & 1.000 & $.616^{*}$ & $.553^{* *}$ & $.648^{* *}$ \\
\hline & & Sig. (bilateral) & .000 & .000 & & .000 & .000 & .000 \\
\hline & & $\mathrm{N}$ & 930 & 930 & 930 & 930 & 930 & 930 \\
\hline & \multirow[t]{3}{*}{ CR-12 } & Coeficiente correlación & $.742^{* *}$ & $.516^{* *}$ & $.616^{* *}$ & 1.000 & $.603^{*+}$ & $.585^{* *}$ \\
\hline \multirow{8}{*}{$\begin{array}{l}\text { Rho de } \\
\text { Spearman }\end{array}$} & & Sig. (bilateral) & .000 & .000 & .000 & & .000 & .000 \\
\hline & & $\mathrm{N}$ & 930 & 930 & 930 & 930 & 930 & 930 \\
\hline & \multirow[t]{3}{*}{ CR-13 } & $\begin{array}{l}\text { Coeficiente de } \\
\text { correlación }\end{array}$ & $.636^{* *}$ & $.421^{* *}$ & $.553^{*+}$ & $.603^{*+}$ & 1.000 & $.488^{*+*}$ \\
\hline & & Sig. (bilateral) & .000 & .000 & .000 & .000 & & .000 \\
\hline & & $\mathrm{N}$ & 930 & 930 & 930 & 930 & 930 & 930 \\
\hline & \multirow[t]{3}{*}{ CR-14 } & Coeficiente correlación & $.789^{*+}$ & $.552^{*}$ & $.648^{* *}$ & $.585^{* *}$ & $.488^{* *}$ & 1.000 \\
\hline & & Sig. (bilateral) & .000 & .000 & .000 & .000 & .000 & \\
\hline & & $\mathrm{N}$ & 930 & 930 & 930 & 930 & 930 & 930 \\
\hline
\end{tabular}

**. La correlación es significativa al nivel 0,01 (bilateral).

Nota: Elaboración propia.

\section{Seguridad.}

Señala la habilidad que tienen los prestadores del servicio para inspirar confianza y credibilidad a los usuarios, los derechohabientes califican el grado de calidad del servicio como malo, existe una desconfianza y poca credibilidad por parte de los usuarios; la correlación bilateral es significativa al nivel 0,01.

En la dimensión de Seguridad, solo el ítem SE-15 obtiene un valor de aceptable, ya que algunas veces el personal médico y administrativo inspira confianza a los pacientes y/o usuarios, lo que genera cierta satisfacción del servicio recibido por los clientes en las clínicas y hospitales en el municipio del Centro, Tabasco (Tabla 5). 
ESTRATEGIAS DE DIFERENCIACIÓN PARA INCREMENTAR LA CALIDAD EN INSTITUCIONES DEL SECTOR SALUD DE TABASCO.

Maı ırinin Ortinnsa Hernándẹ

Tabla 5: Coeficiente Rho de Sperman en forma global y por ítem para la dimensión de Seguridad

\section{Correlaciones}

\begin{tabular}{|c|c|c|c|c|c|c|c|}
\hline \multicolumn{3}{|c|}{ COEFICIENTES } & GGLOB & SE-15 & SE-16 & SE-17 & SE-18 \\
\hline \multirow{15}{*}{ Rho de Spearman } & \multirow[t]{3}{*}{ GGLOB } & $\begin{array}{l}\text { Coeficiente } \\
\text { correlación }\end{array}$ & 1.000 & $.747^{* *}$ & $.744^{* *}$ & $.713^{* *}$ & $.734^{* *}$ \\
\hline & & Sig. (bilateral) & & .000 & .000 & .000 & .000 \\
\hline & & $\mathrm{N}$ & 1860 & 930 & 930 & 930 & 930 \\
\hline & \multirow[t]{3}{*}{ SE-15 } & $\begin{array}{l}\text { Coeficiente } \\
\text { correlación }\end{array}$ & $.747^{* *}$ & 1.000 & $.564^{* *}$ & $.551^{* *}$ & $.567^{* *}$ \\
\hline & & Sig. (bilateral) & .000 & & .000 & .000 & .000 \\
\hline & & $\mathrm{N}$ & 930 & 930 & 930 & 930 & 930 \\
\hline & \multirow[t]{3}{*}{ SE-16 } & $\begin{array}{l}\text { Coeficiente } \\
\text { correlación }\end{array}$ & $.744^{*+}$ & $.564^{* *}$ & 1.000 & $.614^{* *}$ & $.567^{* *}$ \\
\hline & & Sig. (bilateral) & .000 & .000 & & .000 & .000 \\
\hline & & $\mathrm{N}$ & 930 & 930 & 930 & 930 & 930 \\
\hline & \multirow[t]{3}{*}{ SE-17 } & $\begin{array}{l}\text { Coeficiente } \\
\text { correlación }\end{array}$ & $.713^{* *}$ & $.551^{* *}$ & $.614^{* *}$ & 1.000 & $.619^{* *}$ \\
\hline & & Sig. (bilateral) & .000 & .000 & .000 & & .000 \\
\hline & & $\mathrm{N}$ & 930 & 930 & 930 & 930 & 930 \\
\hline & \multirow[t]{3}{*}{ SE-18 } & $\begin{array}{l}\text { Coeficiente } \\
\text { correlación }\end{array}$ & $.734^{* *}$ & $.567^{* *}$ & $.567^{* *}$ & $.619^{* *}$ & 1.000 \\
\hline & & Sig. (bilateral) & .000 & .000 & .000 & .000 & \\
\hline & & $\mathrm{N}$ & 930 & 930 & 930 & 930 & 930 \\
\hline
\end{tabular}

**. La correlación es significativa al nivel 0,01 (bilateral).

Nota: Elaboración propia.

Empatía.

Se refiere a la atención individual y personalizada que proporcionan los prestadores del servicio a los usuarios, los derechohabientes califican el grado de calidad del servicio regular, dependiendo de la hora del servicio la atención va a cambiar, la correlación bilateral es significativa al nivel 0,01.

En la dimensión de Empatía, el ítem EM-24 corresponde a la característica más valorada por los clientes; esta se refiere a que los pacientes y/o usuarios no están satisfechos con la atención individual y personalizada por parte de los servidores en las clínicas y hospitales. (Tabla 6).

Tabla 6. Coeficiente Rho de Sperman en forma global y por ítem para la dimensión de Empatía

\section{Correlaciones}

\begin{tabular}{|c|c|c|c|c|c|c|c|c|c|}
\hline & COEFICI & NTES & GGLOB & EM-19 & EM-20 & EM-21 & EM-22 & EM-23 & EM-24 \\
\hline & GGLOB & $\begin{array}{l}\text { Coeficiente } \\
\text { correlación }\end{array}$ & 1.000 & $.683^{*+}$ & $.610^{* *}$ & $.598^{* *}$ & $.755^{* *}$ & $.745^{* *}$ & $.845^{* *}$ \\
\hline & & Sig. (bilateral) & & .000 & .000 & .000 & .000 & .000 & .000 \\
\hline & & $\mathrm{N}$ & 1860 & 930 & 930 & 930 & 930 & 930 & 930 \\
\hline & EM-19 & Coeficiente de correlación & $.683^{* *}$ & 1.000 & $.475^{* *}$ & $.416^{* *}$ & $.499^{* *}$ & $.512^{* *}$ & $.578^{* *}$ \\
\hline & & Sig. (bilateral) & .000 & & .000 & .000 & .000 & .000 & .000 \\
\hline & & $\mathrm{N}$ & 930 & 930 & 930 & 930 & 930 & 930 & 930 \\
\hline & EM-20 & Coeficiente de correlación & $.610^{* *}$ & $.475^{*}$ & 1.000 & $.384^{*+}$ & $.435^{*+}$ & $.445^{* *}$ & $.519^{* *}$ \\
\hline & & Sig. (bilateral) & .000 & .000 & & .000 & .000 & .000 & .000 \\
\hline & & $\mathrm{N}$ & 930 & 930 & 930 & 930 & 930 & 930 & 930 \\
\hline & EM-21 & Coeficiente de correlación & $.598^{* *}$ & $.416^{*}$ & $.384^{*+}$ & 1.000 & $.587^{*+}$ & $.475^{* *}$ & $.465^{* *}$ \\
\hline & & Sig. (bilateral) & .000 & .000 & .000 & & .000 & .000 & .000 \\
\hline & & $\mathrm{N}$ & 930 & 930 & 930 & 930 & 930 & 930 & 930 \\
\hline & EM-22 & Coeficiente de correlación & $.755^{* *}$ & $.499^{*+}$ & $.435^{* *}$ & $.587^{* *}$ & 1.000 & $.704^{* *}$ & $.625^{* *}$ \\
\hline & & Sig. (bilateral) & .000 & .000 & .000 & .000 & & .000 & .000 \\
\hline & & $\mathrm{N}$ & 930 & 930 & 930 & 930 & 930 & 930 & 930 \\
\hline & EM-23 & Coeficiente de correlación & $.745^{*}$ & $.512^{*}$ & $.445^{* *}$ & $.475^{* *}$ & $.704^{* *}$ & 1.000 & $.645^{* *}$ \\
\hline & & Sig. (bilateral) & .000 & .000 & .000 & .000 & .000 & & .000 \\
\hline & & $\mathrm{N}$ & 930 & 930 & 930 & 930 & 930 & 930 & 930 \\
\hline & EM-24 & Coeficiente de correlación & $.845^{*}$ & $.578^{*}$ & $.519^{* *}$ & $.465^{* *}$ & $.625^{*}$ & $.645^{* *}$ & 1.000 \\
\hline & & Sig. (bilateral) & .000 & .000 & .000 & .000 & .000 & .000 & \\
\hline Rho de Spearman & & $\mathrm{N}$ & 930 & 930 & 930 & 930 & 930 & 930 & 930 \\
\hline
\end{tabular}

**. La correlación es significativa al nivel 0,01 (bilateral). Nota: Elaboración propia. 


\section{CONCLUSIONES:}

A pesar de que desde finales del siglo $X X$ se inicia en México con programas gubernamentales para incrementar la calidad de los servicios de las instituciones de salud pública, pocos cambios se han logrado a la fecha (Sánchez, 2015) En los últimos ocho años Tabasco se ha caracterizado por la baja calidad de los servicios de salud que prestan sus clínicas y hospitales en todo el estado, lo que se ha reflejado en pérdidas humanas

En un trabajo periodístico realizado por Ruth Pérez Magaña del periódico Heraldo de Tabasco y publicado el 18 de diciembre de 2012, señala:" Los cinco hospitales más importantes de Tabasco: Hospital del Niño "Dr. Rodolfo Nieto Padrón", "Gustavo A. Rovirosa Pérez", "Dr. Juan Graham Casasús", de la Mujer y de Salud Mental atraviesan la peor crisis de las últimas décadas debido a que las reservas para seguir operando se agotan en un tiempo máximo de un día hasta una semana. En los nosocomios, el 80 por ciento de las cirugías programadas se han suspendido por la falta de insumos para las intervenciones quirúrgicas informaron los directores de cada una de las unidades médicas..."

El periodista René Alberto López del periódico La Jornada, publicó el viernes 4 de enero de 2013 página 29 "En entrevista, un profesional médico de la Secretaría de Salud, quien pidió anonimato, explicó que el sistema de salud de Tabasco era uno de los más sólidos y llegó a ser el primero del país a partir de 1984, en el gobierno de Enrique González Pedrero. El sector salud de Tabasco, añadió, vivió un auge que se mantuvo hasta la llegada del gobierno de Andrés Granier; "hubo un antes y un después" y hoy se vive una grave crisis".

En una entrevista realizada por Ruth Pérez Magaña del periódico Heraldo de Tabasco, el coordinador de Atención Médica de la Secretaría de Salud (SSA), Bernardo Barradas Ruiz señaló: "La población que acude a los hospitales comunitarios de Tabasco se topa con que no hay médicos en los turnos vespertinos y menos en fines de semana, así como escasez de medicamentos, pues éstos solo se surten en un 80 por ciento en unidades médicas de segundo nivel de atención como son los hospitales asentados en las cabeceras municipales de 10 municipios. Esta falta de personal se da en hospitales de segundo nivel, como los ocho hospitales comunitarios, porque en los 10 hospitales generales se tienen cubiertos todos los turnos" Mencionando que es la falta de recursos económicos lo que impide la contratación de médicos.
Sin lugar a dudas los objetivos que se establecen en el Plan Nacional de Desarrollo y en el Plan Estatal de Desarrollo no se han logrado y por lo tanto las estrategias no han tenido los impactos esperados, lo que nos lleva a vivir los problemas que se señalan en las distintas dependencias del sector salud.

Como podemos observar las Instituciones Públicas Hospitalarias presentan varios retos en la segunda década del siglo XXI, para los cuales tiene que desarrollar un conjunto de estrategias que le permitan posicionarse como los mejores en el estado y en la región, siendo la mercadotecnia quien nos proporciona las herramientas para alcanzar mejoras en la calidad del servicio.

Existen tres grandes retos a los que se enfrentas las instituciones estudiadas: El primero es que una tercera parte de sus derechohabientes cuenta con un nivel de educación de licenciatura, lo que significa una mayor demanda en la calidad de los servicios y al mismo tiempo un personal mejor preparado para poder satisfacer las necesidades de estos derechohabientes. En segundo reto se refiere al equipo, materiales y medicamentos con los que debe contar ya que el $65 \%$ de la población encuestada ocurre a dicho nosocomio solicitando el servicio de consulta externa, lo que nos lleva a pensar en la generación de estrategias financieras que permitan el flujo de efectivo, necesario y suficiente para poder surtir los medicamentos en tiempo y forma y adquirir el equipo de punta para prestar una mejor atención al derechohabiente y el tercero se refiere a la atención de población mayor de 45 años del sexo femenino, por lo que es importante generar estrategias de prevención, buscando con ello una reducción en el número de pacientes que acuden.

Los resultados de la primera parte del cuestionario abre nuevas preguntas en relación al perfil del usuario: ¿Tipos de enfermedades más frecuentes? ¿Periodos en los cuáles existe una mayor demanda? ¿Las veces que ocurre el derechohabientes para la solución de su problema de salud?, entre otras, lo que nos permitiría contar con mayor información para generar una política pública de tercera generación y con ello contribuir a la eliminación de las grandes problemáticas que se tiene en la actualidad. Dicha investigación debería ser inter y multidisciplinaria y con un enfoque holístico.

Estamos conscientes de los grandes problemas económicos que vive el país y en particular el sector de salud pública, pero es necesario repensar esquemas que permitan la innovación y que el cambio permanente sea el común denominador en las instituciones, para ello se requiere que el líder participe de una manera proactiva y que el personal en todos los niveles se sienta integrado al equipo. 
Los datos arrojados en esta investigación sobre la calidad en el servicio de los hospitales son explicados por cinco aspectos principales:

La percepción de los usuarios indica que la calidad recibida no está relacionada con el descuento o pago que realizan los usuarios, no es correspondiente con el servicio recibido por parte de las clínicas y hospitales. Es decir, el descuento o pago no es proporcional al servicio recibido, siendo un factor concluyente para afirmar que no se recibe un servicio de calidad por parte del hospital público en el ámbito de los elementos tangibles.

Respecto a la variable fiabilidad, se obtuvo que la percepción de no recibir un buen servicio desde la primera vez es explicada debido a que el personal del hospital no tiene la capacitación necesaria 0 no tiene el humos (dependiendo de la hora que llegue el derechohabiente) para brindar desde la primera vez, un buen servicio.

En el ámbito de la credibilidad, la percepción de la calidad no se refleja en la eficiencia del servicio del personal. El hecho de que el personal administrativo no cumpla con la entrega de documentos y trámites a tiempo, remite mucha inseguridad en el servicio, y por lo tanto, incrementa la percepción de que no se está recibiendo calidad en el servicio de la clínica u hospital público.

Finalmente, se percibe que los pacientes y/o usuarios no están satisfechos con el servicio recibido medido a través de la variable empatía y se infiere que a medida que no se otorga importancia a las necesidades del usuario se ve reflejada en una menor calidad en el servicio hospitalario.

El descuento o pago realizado por los usuarios no corresponde al servicio otorgado, brindar un mal servicio desde la primera vez, la ineficiencia del personal, incumplir con la entrega de documentos y tramites a tiempo y la insatisfacción de los usuarios por el servicio recibido son los elementos que resultaron con mayores problemas para explicar la baja calidad en el servicio en de las instituciones públicas hospitalarias en el municipio del Centro, Tabasco.

Como podemos observar en cada una de las tablas presentadas en los resultados, de cada una de las variables que establece el modelo, los datos que arrojan nos confirman que la hipótesis del trabajo se comprueba afirmativamente

Ante dichas problemáticas podemos proponer estrategias de desarrollo de productos, es decir, generar nuevos productos y/o mejorar los ya existentes para este mercado, entre las cuales podemos sugerir las siguientes:
- Mejorar las características de los servicios prestados por el factor humano, a partir de un plan estratégico de capacitación y desarrollo de los empleados, tanto operativos como de intendencia y de seguridad.

- Mejorar la calidad en términos de confiabilidad, velocidad y seguridad en la atención al paciente.

- Actualizar sus equipos e instalaciones médicas y crear una imagen de confort que permita al derechohabiente tener los elementos suficientes para que su espera sea agradable. 
A partir de los datos encontrados es necesario que las instituciones públicas de servicios de salud posicionen su "marca" por lo que se proponen las siguientes estrategias de diferenciación (Tabla 7)

Tabla 7: Estrategias de diferenciación competitiva.

\begin{tabular}{|c|c|c|c|}
\hline $\begin{array}{l}\text { Diferenciación del } \\
\text { producto }\end{array}$ & Diferenciación del servicio & Diferenciación del personal & Diferenciación de la imagen \\
\hline $\begin{array}{l}\text { Coordinación con los } \\
\text { distribuidores para la } \\
\text { obtención de equipo } \\
\text { moderno y tecnologías de } \\
\text { punta. }\end{array}$ & $\begin{array}{l}\text { Colaborar con los proveedores } \\
\text { para mejorar muchas } \\
\text { dimensiones que afectan las } \\
\text { características y la calidad del } \\
\text { producto. } \\
\end{array}$ & $\begin{array}{l}\text { Mejorar la apariencia del personal } \\
\text { del hospital. }\end{array}$ & $\begin{array}{l}\text { Copatrocinar promociones y campañas } \\
\text { publicitarias. }\end{array}$ \\
\hline $\begin{array}{l}\text { Introducción de nuevos } \\
\text { equipos o tecnologías } \\
\text { agregando nuevos atributos } \\
\text { o características } \\
\text { innovadoras a estos. }\end{array}$ & $\begin{array}{l}\text { Desarrollar convenios con } \\
\text { universidades y proveedores para } \\
\text { el establecimiento de centros de } \\
\text { investigación para la generación } \\
\text { de patentes tecnológicas } \\
\text { (maquinaria y equipo) para el } \\
\text { servicio médico. }\end{array}$ & $\begin{array}{c}\text { Capacitar al personal (médicos y } \\
\text { enfermeras) para que cumpla en } \\
\text { los tiempos que promete el } \\
\text { servicio. }\end{array}$ & $\begin{array}{c}\text { Desarrollar un plan de publicidad resaltando los } \\
\text { servicios, la tecnología y los casos de éxito del } \\
\text { hospital. }\end{array}$ \\
\hline $\begin{array}{c}\text { Comprar equipos o } \\
\text { tecnologías con mejor } \\
\text { utilidad y funcionamiento } \\
\text { para el beneficio de calidad. }\end{array}$ & $\begin{array}{l}\text { Mejorar la manera en que el } \\
\text { usuario recibe el servicio, la } \\
\text { rapidez, el esmero y la atención } \\
\text { con que se hace la entrega. }\end{array}$ & $\begin{array}{l}\text { Capacitar al personal en general } \\
\text { para generar una cultura de } \\
\text { calidad (hacerlo bien desde la } \\
\text { primera vez). }\end{array}$ & $\begin{array}{l}\text { Mejorar las formas en que la institución } \\
\text { pretende identificarse frente a los usuarios } \\
\text { (identidad). }\end{array}$ \\
\hline $\begin{array}{l}\text { Comprar equipos o } \\
\text { tecnologías con mejor vida } \\
\text { operativa (durabilidad). }\end{array}$ & $\begin{array}{l}\text { Mejorar las instalaciones para } \\
\text { para mejorar las percepciones de } \\
\text { valor por parte de los usuarios. }\end{array}$ & $\begin{array}{l}\text { Capacitar al personal } \\
\text { administrativo para atender de } \\
\text { forma rápida y expedita los } \\
\text { trámites del usuario. }\end{array}$ & $\begin{array}{l}\text { Mejorar la forma en que el usuario percibe a la } \\
\text { institución (imagen). }\end{array}$ \\
\hline \multirow[t]{2}{*}{$\begin{array}{c}\text { Introducción de equipo o } \\
\text { tecnología con seguridad de } \\
\text { uso para inspirar confianza } \\
\text { a los usuarios. }\end{array}$} & $\begin{array}{l}\text { Agregar valor mediante servicios } \\
\text { diferenciados como el precio, } \\
\text { descuento por el servicio. }\end{array}$ & $\begin{array}{l}\text { Capacitar al personal para que: } \\
\text { - Posean la capacidad y } \\
\text { conocimientos necesarios } \\
\text { (competentes). } \\
\text { - Sean amables, respetuosos y } \\
\text { considerados (cortesía). } \\
\text { - Inspiren confianza (credibilidad). } \\
\text { - Proporcionen el servicio con } \\
\text { consistencia y exactitud } \\
\text { (confiabilidad). } \\
\text {-. Atiendan sin retraso las } \\
\text { solicitudes y demoras de los } \\
\text { clientes (capacidad de respuesta). } \\
\text {-. Traten de comprender al } \\
\text { usuario y comunicarse claramente } \\
\text { con él (comunicación). }\end{array}$ & $\begin{array}{l}\text { Incluir anuncios que expresen la personalidad } \\
\text { de la institución y repetirlos en otras } \\
\text { publicaciones como informes, folletos, } \\
\text { catálogos, papel membretado y las tarjetas de } \\
\text { presentación deben reflejar la misma calidad de } \\
\text { la imagen que la institución desea transmitir. }\end{array}$ \\
\hline & & & $\begin{array}{l}\text { Mejorar el espacio físico o ambiente en que la } \\
\text { institución distribuye sus servicios además de } \\
\text { dar una imagen acogedora. }\end{array}$ \\
\hline
\end{tabular}

La implementación de este tipo de estrategias exige un liderazgo transformacional y una administración eficaz y eficiente de los recursos financiero, lo que permitirá a las instituciones públicas del sector salud alcanzar una mayor competitividad a nivel estatal, ya que a pesar de que aparentemente se encuentran en un mercado monopolista al contar con una población cautiva (trabajadores y sus familias), dicho mercado cada vez se ve con una mayor oferta tanto en los servicios hospitalarios y médicos, como en las medicinas con la aparición en la primera década del siglo XXI de los productos similares y los médicos que ofrecen sus servicios a un bajo costo, con lo que el usuario 
decide hacer un pago extra por los tiempos que puede ahorrarse y/o la calidad del servicio que se les ofrece.

Este estudio nos muestra que la calidad del servicio de los hospitales y clínicas del sector público no se ha mejorado a pesar de que existen los instrumentos de política, lo que impacta en que los usuarios no asisten a recibir el servicio a pesar de que tienen derecho, por los que una gran cantidad han decidido por el uso del servicio médico externo, lo que les afecta en su ingreso y empeora su calidad de vida.

\section{REFERENCIAS:}

Casalino-Carpio G. E (2008). Calidad de servicio de la consulta externa de medicina interna de un hospital general de Lima mediante la encuesta servqual. Rev Soc Peru Med Interna, vol. 21 (4).Lima, Perú

Constitución Política de los Estados Unidos Mexicanos, México [Internet]. Recuperado el 10 jun 2017. Disponible en: http//www.dof.gob.mx.

Instituto Nacional de Geografía y Estadística [Internet]. México; INEGI; (2011). Recuperado el10 ene 2017. Disponible en: http://www.inegi.org.mx/

Kotler, P, Amstrong, G. (2013) Fundamentos de Marketing. Ed. Pearson. México; 2013.

Mira J J, Buil A., Rodríguez Marín J, Aranáz J. (1997) Calidad percibida del cuidado hospitalario: adaptación del servqual al hospital público. Gaceta Sanitaria, 11. Alicante, España.

Munuera JL, Rodríguez Al. (2012) Estrategias de Marketing y Teoría de Casos. Ed: Pirámide. Madrid; (349350).

Organización Panamericana de la Salud. (2002) Perfil del Sistema de Servicios de Salud 2da edición, abril 2002. Obtenido de: http://www1.paho.org/hq/dmdocuments/2010 /Perfil_Sistema_Salud-Mexico_2002.pdf
Parasuraman A, Zeithaml VA, \&Berry LL. (1993) Calidad Total de la Gestión de Servicios: cómo lograr el Equilibrio entre las percepciones y las expectativas de los consumidores. Ed. Díaz de Santos. Madrid.

Ríos Fernández N., Samblé Ayala R, Reyes Martínez R (2008). Evaluación de la calidad del servicio al cliente mediante la aplicación del modelo servqual en una clínica del sector salud. CIPITECH. $1^{\circ}$ Congreso internacional de investigación. Cd. Juárez, Chih. México

Salinas R, J.L González, H. (2006) Evaluación de la calidad en servicios de atención y orientación psicológica: conceptos e indicadores en Psicología para América Latina, Psicol. Am.Lat. n.VIII México [Internet] . Obtenido de:

http://pepsic.bvsalud.org/scielo.php?script=sci_arttext \&pid=S1870-350X2006000400014sal

Sánchez, J. (2015) Sistema de salud carece de calidad, dice estudio. Disponible en: http://www.eluniversal.com.mx/articulo/nacio n/sociedad/2015/11/9/sistema-de-saludcarece-de-calidad-dice-estudio

Secretaría de Salud (2010) Sistema Nacional de Indicadores de Calidad en Salud (INDICAS). Dirección General de Calidad y Educación en Salud. Obtenido de http://dgces.salud.gob.mx/INDICASII/

Valenzuela F. (2008) La calidad de servicio en el sector de salud pública: una investigación empírica. Estudios de Administración, vol. 15, №2. Talcahuano Sur, Chile 